\title{
Multilayer networks as embodied consciousness interactions. A formal model approach.
}

\author{
Camilo Miguel Signorelli ${ }^{1,2,3, *}$ and Joaquin Diaz Boils ${ }^{4, *}$ \\ Department of Computer Science, University of Oxford, 15 Parks Rd, Oxford OX1 3QD, United Kingdom. \\ Physiology of Cognition, GIGA institute, University of Liège, 11 Avenue de l'Hôpital, Liège 4000, Belgium. \\ Cognitive Neuroimaging Unit, INSERM-U992, NeuroSpin, CEA, Gif-sur-Yvette F-91191, France. \\ 4 Universidad Internacional de la Rioja, Avda La Paz, 137, Logroño, La Rioja, Spain. \\ * Correspondence: cam.signorelli@cs.ox.ac.uk; joaquin.diaz@unir.net
}

Received: date; Accepted: date; Published: date

\begin{abstract}
An algebraic interpretation of multilayer networks is introduced in relation to conscious experience, brain and body. The discussion is based on a network model for undirected multigraphs with coloured edges whose elements are time-evolving multilayers, representing complex experiential brain-body networks. These layers have the ability to merge by an associative binary operator $\odot$, accounting for biological composition. As an extension, they can rotate in a formal analogy to how the activity inside layers would dynamically evolve. Under consciousness interpretation, we also studied a mathematical formulation of splitting layers, resulting in a formal analysis for the transition from conscious to non-conscious activity. From this construction, we recover core structures for conscious experience, dynamical content and causal efficacy of conscious interactions, predicting topological network changes after conscious layer interactions. Our approach provides a mathematical account of coupling and splitting layers co-arising with more complex experiences. These concrete results may inspire the use of formal studies of conscious experience not only to describe it, but also to obtain new predictions and future applications of formal mathematical tools.
\end{abstract}

Keywords: Autonomy; Conscious experience; Compositionality; Category theory; Mathematical consciousness science; Multilayer network; Radical embodiment.

\section{Introduction}

Mathematical consciousness science is becoming a promising new research field [1]. This novel approach aims to mathematize the phenomenology of conscious experience, focusing either on observable and phenomenal aspects of experience [2-5], or on the mathematical relationships between the physical substrate and consciousness [6-8]. Although the former promises to enlighten our understanding of the formal structures of conscious experience, the latter still presents reminisces of reductionism: consciousness seems reduced to physical electro-chemical neural computations.

In this article, we introduce a general framework to study irreducible brain-body couplings in the context of conscious experience. We first wonder what are specific, but yet simple scalable common principles from cells to assemblies and from assemblies to consciousness and cognition, without reducing higher levels to the lower ones. Our tentative answer invokes compositionality and biological couplings [9], in accord with radical embodiment $[10,11]$. Secondly, to make these ideas precise and model these couplings formally, we translate such principles into conceptual and axiomatic mathematics.

Our hypothetical mathematical structure predicts an irreductive set of experiential brain-body layers and their composition as co-arising with more complex experiences. As a mathematical consequence of decoupling requirements in our mathematical structure, this type of composition also drives causal changes in the structure, one of the most distinguished features of radical embodiment [11]. Therefore, starting from a basic phenomenology of brain and body organization in the context of conscious experience, our mathematical structure imposes mathematical conditions, that we interpreted here as the co-dependence between the brain-body organization and conscious experience.

To target these mathematical relationships, we present an algebraic interpretation of multilayer networks $[12,13]$, where the abstract tensor structure introduced in [14] appears as the simplest way to express the 
semantics of layers as needed. These concepts are introduced across the text with formal definitions and simple examples. For instance, multilayer networks are an extension of Graph theory [12], being the latter the mathematical substrate, explicitly or implicitly, of the most influential neuroscientific models of consciousness $[15,16]$. Therefore, it might be also the simplest and natural choice to generalize mathematical relationships across brain, body and conscious experience. Moreover, multilayer networks present several advantages. It allows to consider signals of different types, simplify seemingly complicated problems within single-network, and describe better emergent phenomena (see examples and applications in [12] and [17]).

All together, our abstract mathematical structures may present theoretical and empirical benefits to reason about experience and its biological substrate. Readers unfamiliar with mathematical structures will be able to grasps these benefits understanding the examples we provide, without any lack of rigour or generality.

\section{Conscious experience and embodiment}

In this section, we introduce the core philosophical concepts and biological principles for our subsequent mathematical formalization.

\subsection{Radical embodiment}

Most scientific models of consciousness fall into one type of monism (e.g. physicalism, dual monism, idealism) [18]. While the so-called "hard problem of consciousness" assumes two opposed substance-ontologies, i.e. two different substances having constant properties and existing each one by itself $[19,20]$. On the one side is conscious experience (qualia), on the other side, the physical body (with its structure, functions, and mechanisms).

A different approach is the metaphysically neutral standing point of phenomenology [21,22]. Within this stream of thought, neurophenomenology and radical embodiment convey a concrete scientific and compelling philosophical model for the biology of conscious experience [11,23-27]. This theoretical framework supposes that conscious experience corresponds to one mode of bodily processes, the lived body, that in turn requires a biological living body. Lived and living body mutually constrains each other, having the former also causal influence in the latter (causal efficacy of experience). Such lived body (experience) can not be reduced to only neural and physical interactions, because experience is assumed to be always embodied into a mesh of more complex biological, social and environmental interactions [28,29]. Moreover, since the lived body and living body are two modes of the same body, it is also wrong to assume that the lived body emerges from the living body (including the brain). This irreductive relationship between lived and living body is better understood as a co-dependence, i.e. in order to define one, we require the other, and vice versa [9].

To formalize the main ideas behind radical embodiment, we require a formal mathematical model that takes into account: i) living properties, such as biological autonomy and embodiment, together with a description of different types of biological interactions, ii) the irreductive nature of brain-body interactions, iii) the irreductive nature of conscious experience, and iv) time-evolving biological systems (dynamics). One would also expect to recover other important features from its mathematical formalization, such as the causal efficacy of conscious experience. In the rest of this article, we approximate some of these requirements following the recent work presented in [9].

\subsection{Compositionality}

The radical embodiment approach urges us to account for the various biological processes that relate to conscious experience, without reducing it to neural and physical systems [9]. The main challenge is doing so in a specific, simple and scalable way.

First, we suggest that conceptual and axiomatic mathematics [30] is the best suited theoretical tool to account for such endeavour. In general, mathematics is ontologically neutral, i.e. the same mathematics can model different phenomena (e.g. similar differential equations can model both physical and biological systems). Specifically, conceptual mathematics, such as Category Theory, axiomatize assumptions, in order to make them explicit and study their consequences. For instance, Category theory studies the relationships of relationships, becoming "the study of patterns of mathematical thought" [31]. It requires precise definitions 
that can rigorously describe systems starting from their relationships, transformations and interactions (processes), instead of accounting for invariant and independent properties (substance). This feature aligns conceptual mathematics with process philosophy [32,33] and presents several advantages to model conscious experience (see arguments in $[5,8]$ ).

Under this framework, ontological existence is only given by interdependent transformations. In our specific case, the lived (mental) and the living (biological) body become related to each other: they are two different modes of the same existence [4].

Secondly, we can model such co-dependence by using the concept of compositionality $[4,5,9]$ (Figure 1). A new whole is a composition of its parts only if the whole has the properties of its parts and vice versa. In other words, the system is non-trivially decomposable [34-36]. Importantly, compositionality accentuates the whole, such that the parts need to be defined by the properties we want to describe in the whole. For example, in category theory [14,37], the composition of two morphisms (processes) $f: x \rightarrow y$ and $g: y \rightarrow z$ in a category, needs to produce another morphism, such as $g \circ f: x \rightarrow z$. The new morphism $g \circ f$ is called the composite of $f$ and $g$. Compositionality forces us to define the parts and the whole simultaneously ${ }^{1}$, demanding a principle which is neither reductionism nor holism (Figure 1).

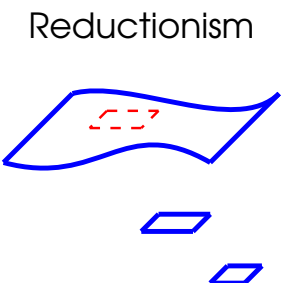

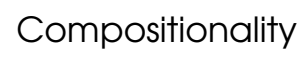

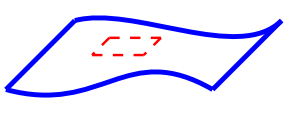

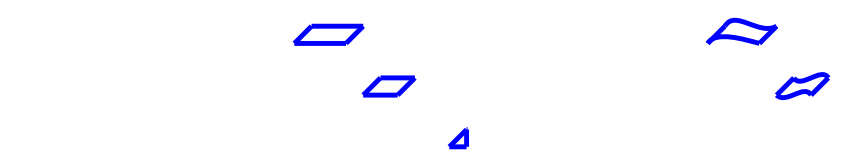

Figure 1. Compositionality. Take the example of oscillatory membranes. In order to analyze them, reductionism will divide the membrane into small pieces (e.g. lipids, proteins, ion channels, etc). In the process, the global properties of the membrane are usually lost. In this example, the whole oscillation. In general, a reductive approach allows us to understand the physical components, but it is unable to recover the whole (usually emergent properties) by the mere description of the parts. Contrary, a compositional approach divides the membrane considering the property of the whole being described. In this example, its capability to oscillate. The smallest component is indeed a group of oscillators. As such, the whole property is always present in the relationship of their parts, and the composition of their parts is much more than the sum of them. Finally, a holistic approach would negate the possibility to explain the membrane oscillation by the mere sum of their parts. Like compositionality, holism claim that the parts of a whole and the whole are interdependent, but differently than compositional approaches, these parts cannot exist without the whole.

\section{Mathematical Methods}

In order to model the main requirements from the previous section, we formally introduce our basic mathematical methods. Familiar readers would like to check the section 8 for advanced material.

\subsection{Multilayer Networks}

Multilayer networks are an extension of the widely used Graph theory. A graph is a tuple $G=(V, E)$, where $V$ is the set of vertices/nodes ${ }^{2}$ and $E \subseteq V \times V$ is the set of edges that connect pairs of nodes (Figure 2A). A graph is a network of interactions, also called single-layer network. Common examples are social networks, transport networks and neural networks given by only one type of interaction (connectivity).

The standard definition of a multilayer network corresponds to a quadruplet $M=\left(V_{M}, E_{M}, V, \mathbf{L}\right)$, where $V_{M} \subseteq V \times L_{1} \times \ldots \times L_{d}$ is the subset of all tuples containing mixed information about the node

1 Compositionality is close to the concept of co-emergence (see [25], p. 431).

2 We will use node and vertice without distinction across the paper. 
and the layers. $E_{M} \subseteq V_{M} \times V_{M}$ is the set of pairs of possible nodes [12], and $\mathbf{L}=\left\{L_{k}\right\}_{k=1}^{d}$ represents a sequence of sets $L_{k}$ of elementary layers/networks. $k$ indicates the aspect of every one of those sets, being $d$ the total number of aspects. The aspects here represent the different interactions between nodes. In our following pictures, these aspects are given by the colour of the edges.

In Figure 2A, we plot a toy example of anticorrelated functional neural networks (red and blue) arising from the interactions of other hypothetical more fundamental layers (yellow and light blue).

The set of edges is partitioned into intra-layer edges and inter-layer edges. Intra-layer edges correspond to the interactions inside layers (e.g. yellow and light blue edges, Figure 2A). The inter-layer edges are those which correspond to interactions between layers (e.g. red and blue edges, Figure 2A). Moreover, in order to account for specific dynamical overlapping nodes (phenomenon called multiplexity [12]), one may consider specific rotation layers, as depicted in Figure 2B (see the Appendix for a formal presentation).

The main advantage of multilayer networks is to decompose what seems to be a whole network into sub-networks that can be described by particular interactions. Biologically, it allows, for instance, the distinction between electrical signals and biological gradients (e.g. map of neurotrasmitter receptors) and/or physiological signals coming from different biological systems. Within a formal and general setup, multilayers simplify the study the coupling and evolution across complex systems of interaction.

As such, layers are mathematical and pragmatic abstractions, like the abstractions we use to analyze the brain as a single network. The first difference is that there is not only one network with only one type of interaction, but many networks and many interactions (given by the colours). It makes a multilayer description more detailed and realistic (e.g. describing neural systems only with action potential signal is a simplification that leaves aside many other relevant interactions that do not involve neural spikes, such as glial interactions). Despite these layers may have biological counterpart, in this work we limit ourselves and focus on describing them only as groups of physiological signals given by senses or organs (Section 4.1). An interesting future development is to link these mathematical layers with concrete biological brain and more general body organization (e.g. [38]).

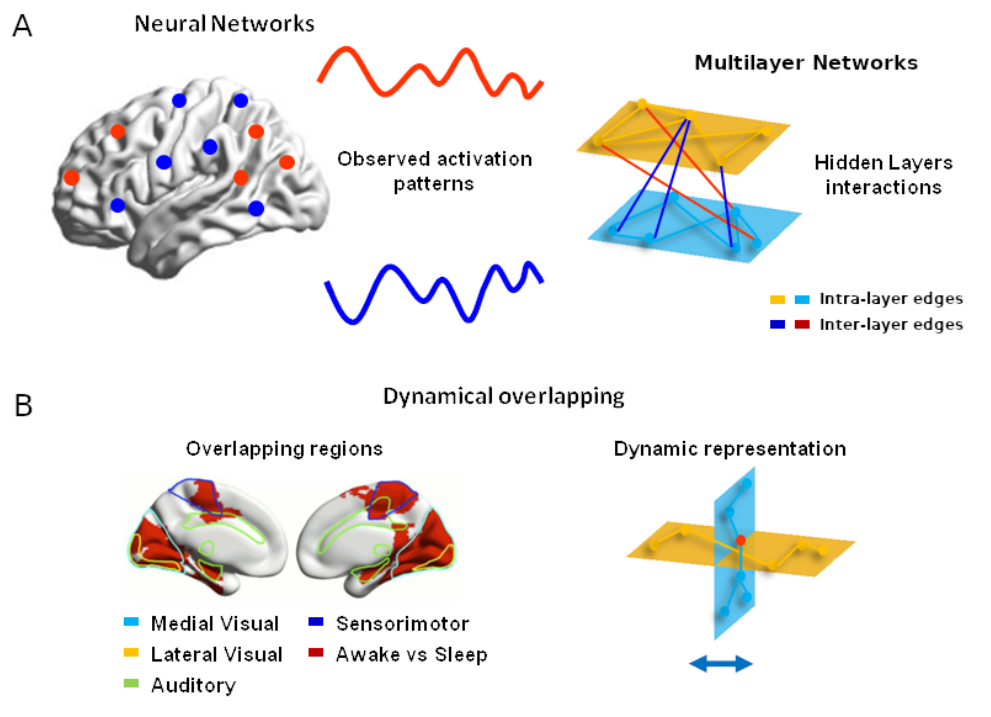

Figure 2. Multilayers for brain networks. (A) Nodes represent a region of interest (right). In this toy example, blue and red colours are families of observed brain networks. Colour lines are their anticorrelated activities (middle). Anticorrelated networks could be the result of more basic or fundamental networks (left, yellow and light blue layers). (B) Example of overlapping regions. The contrast between awake and sleep conditions (right). Different layers of a multilayer network may dynamically overlap. A dynamical representation of this overlapping is given by rotation layers interacting (left). The orange dot corresponds to one overlapping region at a specific time. Adapted from $[9,39,40]$ 


\subsection{Monoidal Interpretation of Multilayer Networks}

If one considers a set of sets of elementary layers, each attached to an aspect into a set of colours and a time index $t$ (not considered as an aspect and formally introduced in section 5), we can give the following formal definition of a multilayer network ${ }^{3}$.

In this setting a multigraph $G$ on a set $V(G)$ of vertices is a multisubset $E(G)$ of pairs of elements of $V(G)$, called edges, with a function $m: E(G) \rightarrow \mathbb{N}$ that calculates the multiplicity ${ }^{4}$ of every edge. In the sequel, every graph will be a multigraph in which loops are not allowed and we colour edges rather than vertices (which gave different models for a multilayer network).

Definition 1. An edge-colored multigraph is a multigraph $G$ together with a (non-necessarily surjective) function col : $E(G) \rightarrow \mathcal{P}(\mathcal{C})$, where $\mathcal{P}(\mathcal{C})$ denotes the set of subsets of the colour multiset $\mathcal{C}$. A graph is said to be monochromatic if all its edges are of the same colour.

Notice that the function $m$ give us the number of times that two specific vertices are connected, while the function col tells us the set of existing colours in those connections. For instance, if we have two layers $G$ and $H$ with two nodes $u$ and $v$, that have $k$ edges between them in $G$ and $k^{\prime}$ in $H$, we obtain $m_{G}(u v)=k$ and $m_{H}(u v)=k^{\prime}$. Similarly, these edges could have different colours, e.g. $\operatorname{col}_{G}(u v)=\left\{c_{1}, \ldots, c_{s}\right\}$ and $\operatorname{col}_{H}(u v)=\left\{c_{1}^{\prime}, \ldots, c_{q}^{\prime}\right\}$. We will make use of $m$ and $c o l$ to express these features rather than the original notation of [12] (see subsection 8.5 for details).

Example 2. Let $M G(n)$ be the set of multigraphs whose vertices are indexed by the set $\mathbf{n}=\{1, \ldots, n\}$. Now we define sets of graphs $(M G(n),+)$ where + is multiset sum, that is, the addition of multiplicities of edges sharing the same vertices.

In the following $(M G(n),+)$ is denoted simply by $M G(n)$. The graphs within are monochromatic and we call them indistinctly layers and graphs in this paper. We identify a layer with a 1-coloured graph, all of the edges within every layer are assigned a single colour $c_{i}$ from a multiset of colours $\mathcal{C}$. We now endow the sets $M G(n)$ with a particular algebraic structure:

Definition 3. A monoid is a tuple $(S, \oplus, \epsilon)$ where $S$ is a set and for every $s, r, t \in S$ :

- $\oplus$ is a total associative binary operation on $S$, that is

$$
(s \oplus r) \oplus t=s \oplus(r \oplus t)
$$

- $\epsilon$ is the identity for $\oplus$, that is

$$
\epsilon \oplus s=s=s \oplus \epsilon
$$

A monoid is said to be commutative if $\oplus$ is commutative, that is, if

$$
s \oplus r=r \oplus s
$$

Example 4. By endowing $M G(n)$ with the so-called overlay operation:

$$
M G(n) \times M G(n) \stackrel{\cup}{\longrightarrow} M G(n)
$$

we obtain a commutative monoid $(M G(n), \cup, \varnothing)$ for which $\varnothing$ is the undirected multigraph with $n$ nodes and no edges and behaves as the identity element (i.e. $G \cup \varnothing=G=\varnothing \cup G$ ). If $n=3$ we calculate $G \cup H$ as

3 This is based on the categorical networks models of [13]. For advanced readers, the content is sketched in section 5 of the Appendix 8.5.

4 A multiset is a set in which some its elements can appear more than once. Then, multiplicity of a multiset is the number of times a certain element appears in the multiset. 

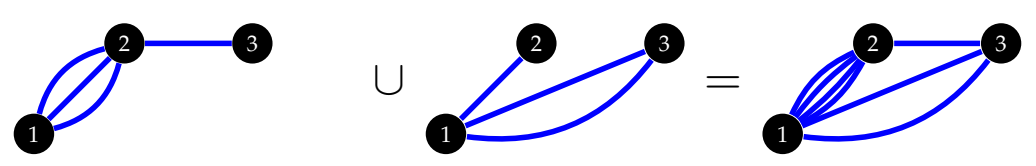

where the multiplicity and colour between node 1 and 2 are given by $m_{G}(12)=3$ and $\operatorname{col}_{G}(12)=\{$ blue $\}$. For the other pair of nodes: $m_{G}(23)=1, m_{H}(12)=1, m_{H}(13)=2, m_{G \cup H}(12)=4, m_{G \cup H}(13)=2$ etc. as well as $\operatorname{col}_{H}(12)=\{$ blue $\}, \operatorname{col}_{G \cup H}(12)=\{$ blue $\}$ etc.

In the following, we need to consider tuples of layers (see below). With this in mind, we construct a colour-indexed tensor product of monoids $M G(n)$, each of them labelled initially with a single aspect (colour). Elements of these tensors are called multilayers. ${ }^{5}$

We put together a number of layers as defined above, where colour could appear in more than one layer if they share the same aspect. The product of $|\mathcal{C}|$ copies of the monoid $M G(n)$ is denoted by $M G^{\otimes \mathcal{C}}(n)$, being $|\mathcal{C}|$ the cardinal of the multiset of colours $\mathcal{C}$ (the cardinality of a multiset is constructed by summing up the multiplicities of all its elements.). The elements of $M G^{\otimes \mathcal{C}}(n)$ are $\mathcal{C}$-tuples of 1-coloured multigraphs with $n$ nodes each. Every graph into $M G^{\otimes \mathcal{C}}(n)$ is called a $|\mathcal{C}|$-coloured multilayer.

The tensor product of sets of multigraphs is constructed here by means of the usual cartesian product of monoids as

$$
\left[M G^{c_{1}} \otimes M G^{c_{2}}\right](n)=M G^{c_{1}}(n) \times M G^{c_{2}}(n)
$$

for the case of $|\mathcal{C}|=2$. In the sequel, we denote $\left[M G^{c_{1}} \otimes M G^{c_{2}}\right](n)$ simply as $M G^{c_{1} \otimes c_{2}}(n)^{6}$.

At the initial time, every layer into a multilayer is identified both with a colour and a position in the tensor product, such as in Figure 3A. They contain edges of a single colour each.

Remark. Recall that the function col in Definition 1 is not surjective, this fact is related to the possibility that some of the layers may have multiplicity zero in every node. That is, we admit graphs $G$ with empty sets $E(G)$ for certain colours or, in other words, we interpret that monochrome layers are multicoloured layers with some missing colours.

\section{Embodiment and composition}

Taking the formal concepts above, we now introduce the biological and experiential interpretations, followed by formal ways to compound the colours of different layers into multilayers. This is given by the operation $\odot$. It accounts for the complexity of brain-body interactions and the irreductive nature of conscious experience.

5 See section 8.1 for a detailed explanation of what exactly we mean by this product of monoids.

6 In [13], the authors explicitly describe the symmetric monoidal structure on the category of coloured network models that allow us to consider coloured multilayers. See the Appendix section for details about this and the construction of the tensor product of monoids. 
A

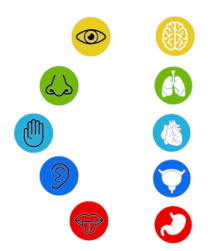

B

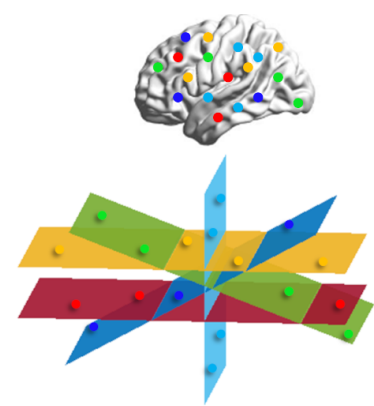

Figure 3. Experiential multilayers for brain and body. (A) Colours represent aspects of experience, while layers the specific instances of those aspects. These aspects are modelled by sensory networks such as vision, audition, etc, but also physiological networks containing the lungs, heart and others. For simplicity, we plot five hypothetical colour layers, but they can be extended to an arbitrary number. These layers are abstractions to visualize brain-body structures. (B) These layers interact forming a multilayer interacting structure. This structure makes certain nodes overlap, having an impact on the observed activity of brain networks. Each circle correspond to a node or region of interest.

\subsection{Biological autonomy and embodiment}

In order to describe biological autonomy and the embodiment of conscious experience, we re-interpret and extend the meaning of layers within a multilayer structure. Following [9], we will assume that each of these layers represents independent or semi-independent biological autonomous systems. Key conditions for biological autonomy are self-production and self-distinction $[25,41]$, such that a biological autonomous system is always operationally closed [42-44].

In the ideal case, layers act as continuous biological membranes (biobranes). Some of these layers might correspond to specific neural networks, while others might account for bodily systems, such as the metabolic system, the cholinergic system, immune system, among others. The elements inside layers (nodes) represent biological processes, cells, neurons, and/or their assemblies, e.g. brain regions and organs such as gut, liver, heart, lungs, etc (Figure 3A). In [9], these systems were thought of as functional, anatomical and metabolic membranes of brain-body organization, acting and reacting as an independent living system (e.g. two amoebas interacting).

Regarding conscious experience, layers bring forth an identity through their agency and convey several isolated or minimal sets of possible experiences. In Figure 3A, for schematic purposes, we associate each colour aspect with one sense, such as layers account for internal (organs) and external (sense) minimal unimodal experiences. Each layer by itself is a closed unity, and the experience becomes embodied by intrinsic internal/external layer definition.

For simplicity, we depict five layers related to the five senses, but nothing restricts our argument to extend it to several other layers of description or contrary, reducing this number. Moreover, each colour represents an experiential aspect that may have an associated set of layers representing specific instances of such aspects of experience. For example, let say that the blue edge colour represents auditory experiences. Then, particular configurations of blue aspect layers would account for specific distinguishable sound experiences like pleasant sound, loud, and noise. Formally, we have a set $L_{b l u e}$ of layers $L_{b l u e^{\prime}}^{1} L_{b l u e^{\prime}}^{2} L_{b l u e^{\prime}}^{3}, \ldots, L_{b l u e}^{j}$, with $j$ the total number of configurations for the aspect represented by the color blue. For instance, in example 4 we have two layer configurations of the same auditory aspect generating a new more complex configuration (the result of the overlay operation). We might have several of these layers configurations depending on the level of detailed experiential description.

The dynamical composition of these layers also has their impact inside the brain, as depicted in Figure 3B. Importantly, it does not mean those body systems and their interactions are reduced to new layers of description in the brain. Since we assume all these layers are autonomous by default, they do not reduce to only neural interactions and do not form any hierarchical organization where neural systems are on top of the others. Quite the contrary, we assume biological layers need a balance to survive and keep interacting [9]. 
In this model, the brain and the body admit a meaningful and non-trivial decomposition into living experiential systems (as mathematically shown in section 5). Notably, to give a first mathematical account, we do not need to specify the nature of these nodes and layers. Here, we recognize that a simple and scalable principle is only the interaction between layers.

\subsection{Composition of layers}

The layers are not by themselves conscious experiences such as we, humans, encounter conscious experience. Indeed, our specific kind of conscious experience will result from the composition of these layers, as we introduce in this section. According to the embodiment interpretation, we treat layers as a minimal bodily organization (living body), as well as a minimal set of possible experiences (lived body). The fact that both are co-dependent is given by the same layer mathematical structure. In our framework, the conscious brain-body acts as an entangled system of experiential layers

Here, we model operations among experiential layers and describe key features of our networks, for that we fix a multiset of colours $\mathcal{C}=\left\{c_{1}, \ldots, c_{d}\right\}$ (from now on every set of colours will be a multiset). We proceed in three steps, endowing $M G^{\otimes \mathcal{C}}(n)$ with three different operations.

Firstly, consider the overlay operation introduced in example 4. This operation is extended to the general case, and as claimed in section 3.2, it licences us to prove that $M G^{\otimes \mathcal{C}}(n)$ are actually monoids. The structure appears as inherited from $M G(n)$ with the overlay operation:

$$
M G^{\otimes \mathcal{C}}(n) \times M G^{\otimes \mathcal{C}}(n) \stackrel{\cup}{\longrightarrow} M G^{\otimes \mathcal{C}}(n)
$$

again with addition for multiplicity.

Example 5. For $n=3, d=3$ with $c_{1}=$ blue, $c_{2}=$ red, $c_{3}=$ yellow:
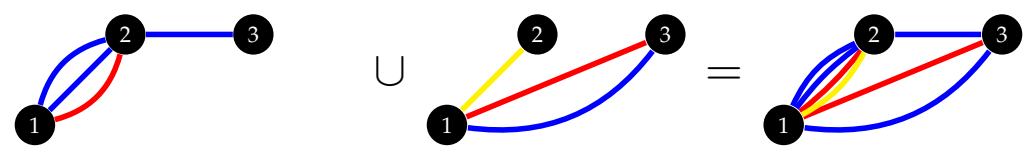

In this case, a 2-coloured layer with two experiential aspects merge with a 3-coloured layer. Both multilayers have the same number of nodes. It accounts for experiences that are experienced as one single experience, without a clear distinction of all the different elements and aspects of such experiences. It brings more and new experiential relationships into a new unified experience. If each colour aspect corresponds to a particular sensory experience, we might have, for instance, blue for auditory experience, red for gustatory and yellow for visual experience. Then, a concrete example may be the construction of experiential things/objects like a red sweet apple that is entirely silent. Although we can distinguish all the qualities of that "apple" (colour edges configurations), we are unable to disentangle them from the apple itself. Therefore, the overlay operation accounts for experiences that are indistinguishable or entirely mixed. The graphical example shows that the overlay operation works for layers with both the same and different experiential aspects (colour edges).

A different restriction conveys the definition of a disjoint union operation:

$$
M G^{\otimes \mathcal{C}}(n) \times M G^{\otimes \mathcal{C}}(m) \stackrel{\sqcup}{\longrightarrow} M G^{\otimes \mathcal{C}}(n+m)
$$

with addition for multiplicity and where $\sqcup$ works as renaming nodes.

Example 6. For $d=4$, one 2-coloured layer and one 4-coloured layer:
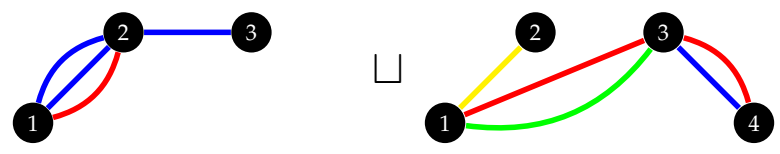

with $n=3$ and $m=4$ respectively we obtain: 

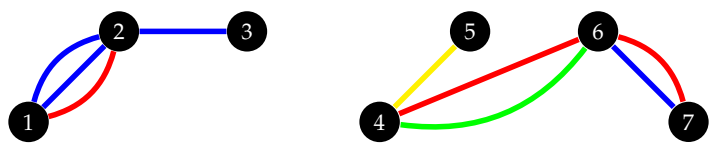

This operation also works for more general cases where layers have different experiential aspects (colour edges). In this specific example, the disjoint operator combines 2-coloured and 4-coloured layers in one new single experience with separate elements inside such experience. Although the new experience is one unified experience (a new multilayer), we can distinguish the elements from original layers, i.e. they are experienced in parallel. Taking the comments about particular configurations from section 4.1, the first multilayer might correspond to an auditory and gustatory experience (e.g. lower sound and sweet), while the second multilayer to auditory, visual, gustatory, and olfactory experience, where now green represent smell. Then, different configurations account for particular instances of such aspects of experience. For instance, the second multilayer might be a high sound, red, bitter and fruity experience. Both multilayers combine to give a new more complex experience. In a unimodal set-up, the disjoint operation also accounts for cases where we experience the same aspect simultaneously (e.g. bitter and sweet when tasting orange marmalade), such that we are able to distinguish them.

Remark. Observe that $M G^{\otimes \mathcal{C}}(n)$ and $M G^{\otimes \mathcal{C}}(m)$ contain, as maximum, all the edges of the same colours since col is not necessarily surjective (that is, not every colour is necessarily present in every layer). This is coherent with the fact that one or more of the factors into a product $M G^{\otimes C}{ }_{(-)}$could be the empty set on edges.

Finally, we define an operation that subsumes our previous $\cup$ accounting for irreducible entangled experiences.

Definition 7. Let $\mathcal{C}$ be a fixed set of colours and $\mathcal{C}_{1}, \mathcal{C}_{2} \subseteq \mathcal{C}$. For every s-coloured layer $G$ in $M G^{\otimes \mathcal{C}_{1}}(n)$ and $q$-coloured layer $H$ in $M G^{\otimes \mathcal{C}_{2}}(m)$ the operation:

$$
M G^{\otimes \mathcal{C}_{1}}(n) \times M G^{\otimes \mathcal{C}_{2}}(m) \stackrel{\odot}{\longrightarrow} M G^{\otimes \mathcal{C}_{1} \cup \mathcal{C}_{2}}(n+m-p)
$$

produces a new $(s+q)$-colored layer $G \odot H$ in $M G^{\otimes \mathcal{C}_{1} \cup \mathcal{C}_{2}}(n+m-p)$ where $p=|V(G) \cap V(H)|$.

Taking our example of $G$ and $H$ as layers, we now have $m_{G \odot H}(u v)=k+k^{\prime}$ and $\operatorname{col}_{G \odot H}(u v)=$ $\left\{c_{1}, \ldots, c_{s}, c_{1}^{\prime}, \ldots, c_{q}^{\prime}\right\}$, where some of the colours may repeat.

Example 8. For $n=3, m=4, s=q=2$ and $p=3$ :

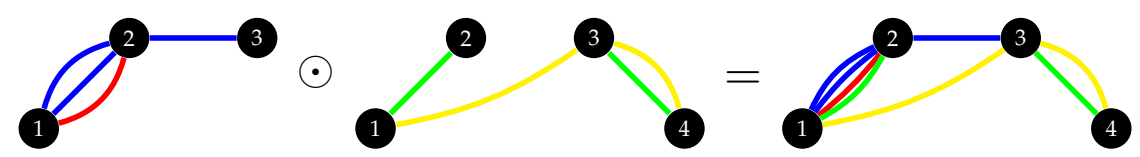

Now we combine all the notions introduced so far. Keeping the colour corresponding to different sensory modalities ${ }^{7}$, the composition is given by $\odot$ combines multilayers in order to account for an experience that combined all modalities, as richer dimensions of complex experiences. The new composition is an edge-coloured multilayer with multiple aspects. In example 8 , the result of $\odot$ gives a new edge-coloured multilayer with four aspects (e.g. audition, taste, smell and vision), such that this particular $\odot$-direct product of multigraphs is chosen as a way of avoiding any information lost from original layers, whenever forming new multilayers.

That is, several experiential layers compose to model new simultaneous experiences or multi experiential layers where some elements may be distinguishable and others not (disjoint and overlay operation respectively).

7 Please, bear in mind it can be generalized to more aspects. 
This new experience is unified but given by both, parallel and combined experiences accounting for various experiential elements at the same time. Blue layers are bitter or sweet according to their configurations, different red layers correspond to particular smells, and we merge layers to account for complex combinations of new experiences. This operation becomes a more general case, from which we emphasise later the possibility of obtaining a meaningful decomposition (see section 5).

Note that, in the sense of [12] and section 3.1, all the three operations above do not generate any inter-layer edge among layers but rather the intra-layer edges of a $(s+q)$-aspect layer from previous $s$-aspect and $q$-aspect, in the case of $\odot$. In other words, our layers compose in such a way that the intra-interaction of one layer become part of the intra-interactions of the other layer, and vice versa.

This is a relevant difference with other types of multilayers since it accounts for the embodiment of different systems forming a new systemic whole. This mathematical property is important, not only because it describes the difficulty to isolate brain-body interactions under states of conscious experience, but also because it may tell us how monochromatic unisensory layers combine to construct richer experiences.

One can identify a single $\left|\mathcal{C}^{\prime}\right|$-coloured layer into a $|\mathcal{C}|$-coloured multilayer for $\mathcal{C}^{\prime} \subseteq \mathcal{C}$ from the interaction through $\odot$ among all the different layers. Roughly speaking, $\odot$ shows the way by which a multilayer can combine minimal experiences (colour layers) by the process of merging layers.

Remark. Observe that, whenever $n=m$ and $\mathcal{C}_{1}=\mathcal{C}_{2}=\mathcal{C}, \odot$ reduces to the internal operation $\cup$, that is, $\odot$ appears formally as a generalization of $\cup$. In fact, we may have considered $\odot$ as the internal operation for that the different $M G^{\otimes C}(n)$ become monoids. In this case, one should note that not only $n$ is fixed but also $\mathcal{C}$, where the latter appears as a fixed set of colours where some of the elements could be missing.

\section{Dynamical interaction of layers}

The final requirement to model radical embodiment is its dynamical evolution. The addition of this feature gives a richer account for different states and contents of conscious experience. Moreover, it allows us to recover the causal efficacy of conscious interactions.

\subsection{Rotation layers}

Rotation layers are mathematical abstractions to account for functional and dynamical overlapping regions and networks [45,46] (see examples in Figure 2B, 3B, and 4). This dynamical configuration is described by multilayers with a rotation angle. This angle is time-indexed, making every layer dependent on a time parameter. It allow us to formalize the intuition of agency or will to interact with other layers, such as bigger angles represent greater willingness to interact. We formally introduce a notion of a multigraph endowed with a rotation angle as follows:

Definition 9. A rotation graph is a pair $[G, \alpha]$ where $G$ is an edge-coloured multigraph and $\alpha \in[-\pi / 2, \pi / 2]$.

Every rotation graph in our model is now a layer with the ability to rotate. In time $t=0$ they are in parallel non-interacting position, such as exhibited in the rightmost picture of Figure $3 \mathrm{~A}$, moreover, at that initial time $t=0$ every layer contains a unique colour. Given an interval $T \subseteq \mathbb{R}^{+}$, we consider angles in the form of continuous functions $\alpha: T \rightarrow[-\pi / 2, \pi / 2]$. Then:

Definition 10. Two rotation graphs $[G, \alpha]$ and $[H, \beta]$ interact in a time $t$ if $\alpha(t)$ and $\beta(t)$ have a different sign, considering zero as a sign in itself, and $|\alpha(t)|+|\beta(t)| \geq \pi / 2$.

Multigraphs are constructed with as many edges of each colour (between any pair of distinct vertices) as present in the involved multigraphs. This is done by several applications of $\odot$.

Since we are interested in networks for which the interaction in definition 10 does actually take place, all the operations for multigraphs in section 4.2 are subject to that condition. That is, they are partial operations. Partiality means that $\oplus$ is an internal operation for a set subject to some condition that has to be specified, in our case we define the sets

$$
R M G^{t}(n)=\{\text { rotation graphs }[G, \alpha] \text { with }|V(G)|=n \text { and rotation angle } \alpha(t)\}
$$


by setting $R M G^{0}(n)$ to contain only 1-coloured multigraphs when no interaction has taken place.

The above allows us to consider multilayers with rotation on its constituent layers. Every element of $R M G^{t}(n)$ is then called a rotation layer or simply a layer. A rotation multilayer network for multigraphs with coloured edges is

$$
\left[R M G^{t}\right]^{\otimes \mathcal{C}}(n)
$$

i.e, a product of $|\mathcal{C}|$ copies of $R M G^{t}(n)$.

Every element of $\left[R M G^{t}\right]^{\otimes \mathcal{C}}(n)$ is called a $|\mathcal{C}|$-coloured rotation multilayer or simply a $|\mathcal{C}|$-coloured multilayer. As above, we consider a more general form of coloured rotation multilayer as

$$
\left[R M G^{t}\right]^{\otimes \mathcal{C}_{1}}\left(n_{1}\right) \times \ldots \times\left[R M G^{t}\right]^{\otimes \mathcal{C}_{k}}\left(n_{k}\right)
$$

Analogously, we consider an internal operation on every $\left[R M G^{t}\right]^{\otimes \mathcal{C}}(n)$ that makes use of the previous operator U:

Definition 11. For every interacting pair of layers $[G, \alpha]$ and $[H, \beta]$ in $\left[R M G^{t}\right]^{\otimes \mathcal{C}}(n)$ we define

$$
\left[R M G^{t}\right]^{\otimes \mathcal{C}}(n) \times\left[R M G^{t}\right]^{\otimes \mathcal{C}}(n) \stackrel{\cup}{\longrightarrow}\left[R M G^{t}\right]^{\otimes \mathcal{C}}(n)
$$

where

$$
[G, \alpha] \cup[H, \beta]=[G \cup H, \min (\alpha(t), \beta(t))]
$$

This operation endows $\left[R M G^{t}\right]^{\otimes \mathcal{C}}(n)$ with the structure of a partial monoid (see section 8.2) whose identity is given by $[\varnothing, \pi / 2]$, where $\pi / 2$ means the undirected multigraph with $n$ vertices and no edges and $\pi / 2$ is the constant function. ${ }^{8}$

Now, the $\odot$ operation in the non-rotation case is extended to the rotation (rotation operators are denoted with bold and slightly bigger symbols). We take into account that this operation is partial since it works only in the case that the involved layers do interact. In a time $t>0$ rotation layers compose as follows:

Definition 12. For every interacting $s$-coloured layer $[G, \alpha]$ in $\left[R M G^{t}\right]^{\otimes \mathcal{C}_{1}}(n)$ and $q$-coloured layer $[H, \beta]$ in $\left[R M G^{t}\right]^{\otimes \mathcal{C}_{2}}(m)$

$$
\left[R M G^{t}\right]^{\otimes \mathcal{C}_{1}}(n) \times\left[R M G^{t}\right]^{\otimes \mathcal{C}_{2}}(m) \stackrel{\odot}{\longrightarrow}\left[R M G^{t}\right]^{\otimes \mathcal{C}_{1} \cup \mathcal{C}_{2}}(n+m-p)
$$

produces a new $(s+q)$-coloured layer containing a graph

$$
[G, \alpha] \odot[H, \beta]=[G \odot H, \min (\alpha(t), \beta(t))]
$$

in $\left[R M G^{t}\right]^{\otimes \mathcal{C}_{1} \cup \mathcal{C}_{2}}(n+m-p)$ where $p=|V(G) \cap V(H)|$.

Differently than in the initial time, layers are not tied to any particular position in the tensor product. The final number of vertices after a long product such as

$$
\left[G_{1}, \alpha_{1}\right] \odot \ldots \odot\left[G_{k}, \alpha_{k}\right]
$$

comes from an application of the Inclusion-Exclusion Counting Principle (see section 8.7 for details).

\subsection{Content structure for dynamical experiences}

In the dynamical case, every rotation layer mixes colours as in the previous section 4.2. However, this new mixing involves the notion of coupling: the $\odot$ commutative operator preserves the individuality of layers'

8 The operator $\cup$ is also useful to check that our previous 1-coloured sets $R_{M G}(n)$ are partial commutative monoids themselves. 
colours at the cost of losing their angle independence, (i.e. its inner dynamical activity). We represent this dynamical coupling and possible transitions by the picture in Figure 4A. Note that in the rotation monoids new edges (and possibly new colours) appear in a layer only after the interaction of two layers where the union $\mathcal{C}_{1} \cup \mathcal{C}_{2}$ is the multiset colouring $G \odot H$.

A
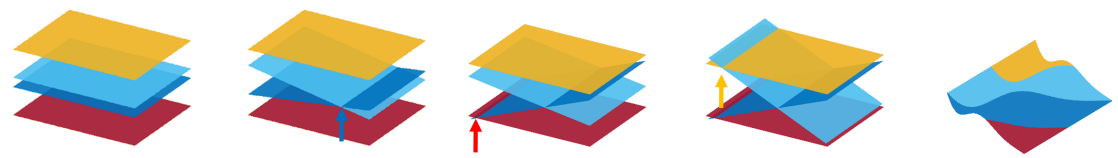

First Transition

Second Transition

Third Transition

B
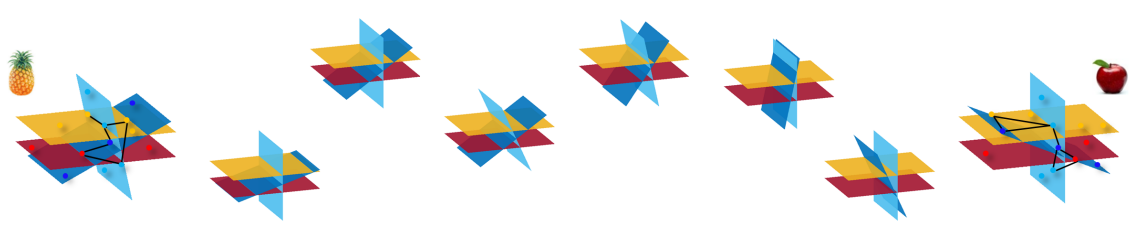

Figure 4. Consciousness interaction and phenomenology. (A) Different layers may dynamically interact generating different transitions and local-global new multilayers. First transitions may correspond to wakefulness, followed by transition representing phenomenal experience, awareness, and other transitions related to more complex phenomenal content structure. After all transitions and compositions, we end up with one multi-colour layer. (B) Different dynamical layer configurations may co-arise with the phenomenology of conscious experience. Here, an example of layer configuration from the content of pineapple to apple, and their dynamical changes.

In this picture, edges whose constituent nodes appear both in more than one layer (coloured more than once) are allowed. They are called coupling edges and admit an underlying coupling graph containing the basic configuration of the system. These nodes should be seen as part of a core structure of the brain-body. In the extreme case that a coupling edge is coloured with all colours into $\mathcal{C}$, we have a pair of nodes that participate in every conscious operation that such a system might have. As a purely mathematical consequence of our formalization, the existence of a core structure for conscious experience appears as given by nodes that are coloured by all coloured edges.

Postulate 1. Core structure. A pair of nodes that are coloured by coupling edges with all colours in $\mathcal{C}$ define a core structure for conscious experience. The core structure for a multilayer network is the subset of all nodes such that whenever they are connected, the connection consists of at least one colour edge of all colours. Formally:

Definition 13. Let $\mathcal{C}=\left\{c_{1}, \ldots, c_{d}\right\}$ a set of colors, $\mathbf{L}=\left\{L_{k}\right\}_{k=1}^{d}$ a set of layers and $[G, \alpha] \in\left[R M G^{t}\right]^{\otimes \mathcal{C}}(-)$ a rotation graph from the interaction of rotation graphs in $\mathbf{L}$ and for which every color in $\mathcal{C}$ is present at least once. Its core structure is the subset $V($ Core $) \subseteq V(G)$ where Core is a subgraph of $G$ such that for every $(u v) \in E($ Core $)$ one has col $_{\text {Core }}(u v)=\left\{c_{1}, \ldots, c_{d}\right\}$.

Example 14. For $d=5$ with $c_{1}=$ blue, $c_{2}=$ red, $c_{3}=$ yellow, $c_{4}=$ green, $c_{5}=$ celeste and the following multigraph $G$ :

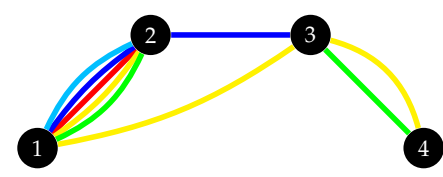

its core structure would be the set $\{1,2\} \subseteq V(G)$.

These nodes might correspond to observed brain-body regions whose contribution to the conscious experience is diverse (section 6). In our model, the core structure is a consequence of conscious processes interacting, as new coupling edge activities appear. This is different from other models claiming core 
structures for consciousness. In such models the core structure is the effective cause, or from which conscious experience would emerge, which is not our case here (see section 6 for discussions).

Furthermore, the content of conscious experience is given by dynamical configurations of rotation layers interacting and composing whole systems. One toy example is shown in Figure 4B. Regions in the coloured layers are part of the different processes according to their network affiliation at a given time. If they form part of several layers, they define a dynamical core structure (black edges Figure 4B) that might co-arise with the phenomenal and concrete content of conscious experience.

Postulate 2. Phenomenal content. The aspects, configurations and degree to which layers interact with each other co-arise with the dynamical structure and content of experience. Formally:

- if $u v$ is an edge in a monochrome layer, the value of $\operatorname{col}_{G}(u v)$ accounts for a single colour relating to a unisensory experience (e.g. gustatory layers in which each configuration correspond to salty, bitter, sweet, etc.)

- if $u v$ is an edge in a multichromatic layer, the value of $c o l_{G}(u v)$ may account for a subset of the more general set of diverse experiences (e.g. salty, green, loud, etc, altogether).

Informally, an interaction for which $|\alpha(t)| \geq \pi / 4$ or $|\beta(t)| \geq \pi / 4$ is viewed as a strong interaction, while a situation such as $|\alpha(t)|=\pi / 2$ and $|\beta(t)|=0$ is interpreted as a total interaction for $\alpha$ while $\beta$ stays in a non-interacting position or, geometrically, as $\alpha$ being perpendicular while $\beta$ stays parallel. The in-between configurations represent the dynamical evolution of these layers. For example, in Figure 4B, the blue layers change the position while the others remain fixed. These changes inform about different content states. The experience evolves in one or another form, the content is about one or another element, the whole system feels one or another feeling. The structure and content of experiences not only depend on the degree (strong, total, etc), aspects and configurations of layers interacting, but this structure and content of experience also affect and defines the degree, aspects and configurations of the interactions on which they depend, i.e. these lived and living layers indeed co-determine each other definition.

\subsection{Splitting layer experiences}

After showing how layers combine we address the issue of splitting layers from a certain configuration. We propose a way to reduce back a coloured multigraph to a multiset of single-coloured graphs. For this purpose, we take a particular decomposition of graphs for which we recover all the nodes involved in the interactions. As will be seen, we compose using $\odot$ but decompose using $\cup$.

Once we have at our disposal infinite sets of rotation graphs in the form $\left[R M G^{t}\right]^{\otimes \mathcal{C}}(n)$, we can isolate a particular subset in the initial time giving a formal account of the experiences referred to above and thus performing our model for brain-body. This will consist of a set of sets of layers, monochrome in time zero and ready to merge through $\odot$, producing richer multicoloured experiences. We will depict a way to split those experiences into a set of indecomposable experiences which turns out to be unique up to rotation angle.

Definition 15. Consider a fixed multiset $\mathcal{C}=\left\{c_{1}, \ldots, c_{d}\right\}$. Let be Biobrane $=\left\{B_{k}\right\}_{k=1}^{d}$ where:

- every $B_{k}=\left\{B_{k}^{i}\right\}_{i=1}^{m_{k}}$ is a set of layers with cardinality $m_{k}$ and

- every multigraph $[G, \alpha]$ in a layer $B_{k}^{i}$ belongs to $\left[R M G^{0}\right]^{c_{k}}\left(n_{i}\right)$ where $n_{i}$ is the number of vertices.

That is, Biobrane is the initial multilayer of experiences (minimal indecomposable and irreducible set of experiences), where no interaction has been taken place. In a certain time $t>0$ interacting layers merge to obtain multicolored multigraphs in $\left[R M G^{t}\right]^{c_{1} \otimes \ldots \otimes c_{d}}(p)$ where $p$ appears as an application of the Inclusion-Exclusion Principle referred to in section 5.1. For this purpose let $\left\{\left[G_{1}, \alpha_{1}\right], \ldots,\left[G_{m}, \alpha_{m}\right]\right\}$ be the set of irreducible layers or atoms such that

$$
[G, \alpha]=\bigodot_{j=1}^{m}\left[G_{j}, \alpha_{j}\right]
$$


where every $\left[G_{j}, \alpha_{j}\right]$ belongs to a certain $B$ into Biobrane and note that $[G, \alpha]$ lives in $\left[R M G^{t}\right]^{c_{1} \otimes \ldots \otimes c_{d}}(n)$ rather than Biobrane.

Now, given these $\left[G_{j}, \alpha_{j}\right]$ in Biobrane, there exists a set $\left\{\left[H_{1}, \beta_{1}\right], \ldots,\left[H_{m}, \beta_{m}\right]\right\}$ such that

$$
[G, \alpha]=\cup_{j=1}^{m}\left[H_{j}, \beta_{j}\right]
$$

where every $\left[H_{j}, \beta_{j}\right]$ belongs to a certain $\left[R M G^{t}\right]^{c_{k}}(n)$ such that $t>0$. Our choice of a decomposition can be checked in its details in section 8.8. Some essential features of this decomposition are:

1. every $\left[H_{j}, \beta_{j}\right]$ is an atom for a monoid $\left[R M G^{t}\right]^{c_{k}}(n)$ since every graph was constructed out of atoms in Biobrane,

2. edges in every initial layer appear together to the end in every further combination and

3. given a set of atoms in Biobrane, the decomposition described is unique up to rotation angle.

Example 16. We take, for the sake of clarity, usual sets (that is, no multisets) $B$ in Biobrane. Merge two graphs by using $\odot$ as:

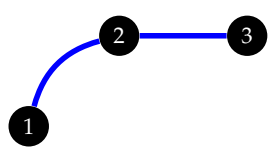

\section{$\odot$}
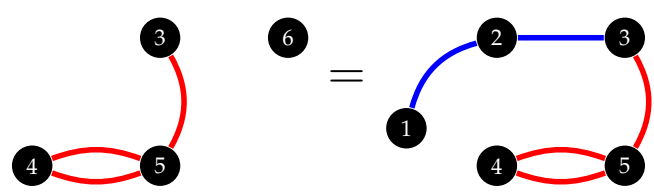

where the left-hand term is $\left[G_{1}, \alpha_{1}\right] \odot\left[G_{2}, \alpha_{2}\right]$ for interacting:

- $\left[G_{1}, \alpha_{1}\right]$ in $\left[R M G^{0}\right]^{c_{1}}(3)$

- $\left[G_{2}, \alpha_{2}\right]$ in $\left[R M G^{0}\right]^{c_{2}}(4)$

and $c_{1}=b l u e$ and $c_{2}=$ red. Now, starting from the merged multigraph, we make use of $\cup$ to split it as:

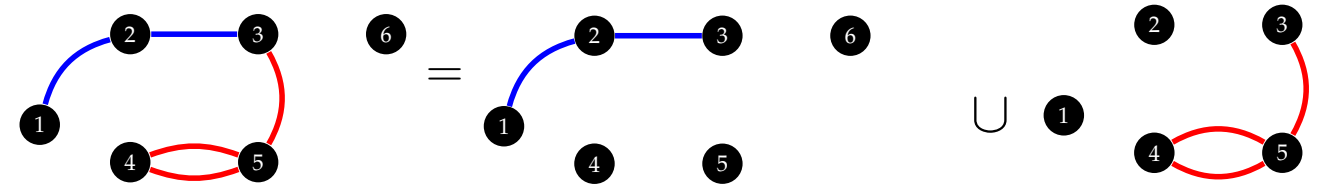

where the right-hand term is $\left[H_{1}, \beta_{1}\right] \cup\left[H_{2}, \beta_{2}\right]$ for non-interacting:

- $\left[H_{1}, \beta_{1}\right]$ in $\left[R M G^{t}\right]^{b l u e}(6)$

- $\left[H_{2}, \beta_{2}\right]$ in $\left[R M G^{t}\right]^{\text {red }}(6)$

with $t>0$ and $\left|\beta_{1}\right|+\left|\beta_{2}\right|<\pi / 2$. Observe that it is satisfied

$$
\left[G_{1}, \alpha_{1}\right] \odot\left[G_{2}, \alpha_{2}\right]=\left[H_{1}, \beta_{1}\right] \cup\left[H_{2}, \beta_{2}\right]
$$

\section{Implications and discussion}

Across previous sections, we introduced a simple algebraic model of layers and multilayers representing experiential and biological brain-body systems. In the beginning, each system is constituted by a one-colour graph. These multilayers can interact in various ways, via composition, disjoint union, and also via a rotation mechanism. This framework allows us to reason about the brain-body organization and describe key configurations related to human conscious (lived body) and unconscious (living body) experiences (Figure 4). 


\subsection{Irreductive experiences}

In our formalism, monochrome layers represent both minimal experiences and living bodily systems, such as richer forms of the former are given by the coupling of the latter. Indeed, conscious and unconscious experiences are described as two modes or configurations of the same system, here represented by a group of layers in a multilayer structure. Therefore, conscious experience would co-arise with the interaction of these biological layers, such that intra-edges of one layer become new intra-edges in a richer layer via the $\odot$ operation.

It is important to notice that the lived experience (mind) and living experience (body) do not convey an identity theory. Identity theories claim that conscious experience either emerge from the biological configurations of the lived body or identically corresponds to those configurations, following one to one relationships. Therefore, explaining a particular conscious experience is equivalent to explaining the particular configuration, either in the brain or the full body. This approach is ultimately intractable since it is impossible to track simultaneously all the elements in such systems and consequently, determine a unique state of it (see $[47,48])$.

Contrary, radical embodiment would claim that lived body is neither reduced to, nor emerge from the living body, but both are dynamical modes of the same existence. This approach does not imply that describing all "free parameters" of a living system will end into a unique description of conscious experience. Quite the contrary, the embodiment implies that this is never the case. Degeneracy of physiological systems (the fact that multiple micro-configurations end up in similar global or macro-configurations) and the irreductive character of experiential decompositions do not allow such methodological reduction. In radical embodiment, the description of the system and experiences are given by a mutual or contextual constrain, i.e. the decompositions are chosen together with the experiences we want to describe, and therefore, the decomposition is co-dependent with such experiences. Then, in our mathematical model, these configurations become irreducible to each other. Therefore, conscious lived experience does not emerge from these interactions. It is just described as a more complex composition of layers, such that we can incorporate the intuition of the transcendental character of experience: "lived experience is always already presupposed by any statement, model, or theory, and the lived body is an a priori invariant of lived experience" [25].

\subsection{A biological neuroscience of consciousness}

Layers model biological autonomous systems and irreductive experiential units that bring an identity through the sense of the environments, significance and value of their interactions (self-making) [24]. These systems are "minimally decomposable", or nondecomposable at all [11]. It means that layers represent global processes that subsume their components such that they are nonseparable. For example, local neural activity might emerge from the whole dynamic patterns of large-scale activity as much as the whole emerges from their local components [25]. The distinction between parts and whole vanish (section 2.2). Moreover, our mathematical results point out that these layers are contextual decompositions (section 5.3), but not ontological ones. In other words, we emphasise the irreducibility of relations where layers become patterns that exist in relationship with each other: all are equal interacting processes and none has ontological primacy over the others.

This ontological neutral approach, however, presents several advantages. From very simple, yet scalable principles, we obtain several important results. For example, the postulate 1 is a consequence of the formal treatment and deeper assumptions. Other models of consciousness, to more or less extend, also claim core structures. Some examples are the global workspace (GW) [49], the global neuronal workspace (GNW) [50], the dynamical core [51], and the memory evolutive neural systems (MENS) [52], among many others. All these models treat conscious experience as emerging and reduced to the core structure, while this structure is postulated as a key initial assumption. One exception is MENS, where the core structure is also a consequence of mathematical formalization. In radical embodiment, however, the core structure arises as a consequence of embodiment and it is not an initial assumption, such as in GNW.

The second postulate is akin to the relationships between biological substrates and the phenomenology of conscious experience presented in the dynamical core and integrated information theory (IIT). For example, 
through the definition of the "maximum irreducibility of cause-effect repertoires" and their "complexes". Concretely, the variety of layers and their couplings would trigger the complex configurations and global brain dynamics observed in awake conditions (e.g. functional connectivity patterns). Nevertheless, the radical embodiment is neither an identity theory (section 6.1) nor a computational theory reducing body interactions to only one type of neural interactions, such as IIT seems to be.

Radical embodiment and our formal model is a general biological theory for the neuroscience of consciousness that might indeed subsume others (e.g. GNW, IIT, etc). In detail, once the reciprocal actions are in place through composition and coupling layers, each layer receives the influence of the other via the new coloured edges. Because we impose these interactions need to have biological meaning (e.g. metabolic exchanges between layers), each layer might care about its and others processing, as part of its biological requirements and the others biological requirements, which can directly affect them. It should be seen in direct analogy to how any living being acts and reacts to different types of contexts, ensuring its survival. In other words, these virtual biological layers ultimately act as biological entities and not only "informational units". As such, any biological entity, after interactions with their pairs, also need periods of non-interaction (e.g. by means of sleep or rest). Basically, the whole system and its parts try to keep their balance, coupling and decoupling from time to time, as part of their own biological demands.

These decouple correlate with a reduction of awareness even under global awake conditions. Interestingly, this reduction of brain activity seems to be present between two moments of consciousness [53,54], as some dynamical brain analyses associated with unconsciousness predict during conscious resting-states [55]. Our theoretical model explains this reduction by the natural and biological need to go back to intrinsic dynamics of splitting layers. In other words, this reduction is due to decoupling of layers, or the recovery of intrinsic layers dynamic against the detriment of interactions that would interfere with those intrinsic layer dynamics. In our model, all these imply that if one process layer disappears, the awareness associated with that process also disappears.

\subsection{Causal experiential efficacy}

As a mathematical consequence of the irreductive nature of conscious experiences, such experiences also appear as triggering causal effects in the layer system. These effects are given by the splitting layer constrain, imposing a type of interacting memory after layers composition. The new nodes after the splitting process represent a memory of the layer-interaction that we interpret as a causal effective action or sub-emergent action of conscious activity into the biological substrate [56].

To detail the consequences of section 5.3, we note that, although splitting layers in $\left[R M G^{t}\right]^{\otimes \mathcal{C}}(n)$ is also possible, we are not reaching the same original picture we had at time $t=0$. Indeed, we are recovering all the edges in separate layers at the cost of carrying a copy of every node that is found in the maximum-coloured multilayer from which they arise. That is, after the splitting process every monochrome layer appears with the same edges as it had, but also carries a copy of the vertices in the layers with which interaction has taken place. With the notation of section 5.1, such a new number of vertices is the result of an application of the Inclusion-Exclusion Counting Principle. In short, we make use of the operation $\odot$ for merging layers but in the process of splitting them back, they appear as a U-product.

This mathematical constrain fits well with the idea of causal experiential efficacy, sometimes called downward causation [25,57]. The multilayer structure has causal effects as a second-order topological and contextual constrain in the individual layers that constitute the whole system. Importantly, in our mathematical treatment, this is not an "external force" that acts on the biological system, but the mathematical consequence of interconnectedness or relatedness among layer processes after composing and splitting.

In summary, the model does not only predict that layers involved in conscious experience compound in such a way that the intra-interactions of one become intra-interactions of the others, but also that the interaction associated with conscious experience have an impact in the biological substrate. 


\section{Conclusions}

Across these pages, a simplified version of radical embodiment was introduced and their main concepts were generalized by using symmetric monoidal category for multigraphs. We formally defined biological experiential layers, their interactions, and study their mathematical consequences. According to the chosen set-up, the main results of these discussions are the definition of the $\odot$ operation, modelling the composition of experiential layers, and the implications of the uniqueness (up to rotation) of the layer decompositions. Moreover, we have recovered a few extra ingredients, such as core structures for conscious experience, dynamical content and causal efficacy of conscious interactions.

Future developments are expected as long as the characterization of multiple features of the theory is possible. Some concrete research lines are a) giving a concrete phenomenological and biological account for specific layers configurations, b) finding an algebraic and categorical way to describe the graph contraction among the splitting of rotation multilayers, discussed in section 5; c) study new features arising after going back the multilayers to parallel, non-conscious landscapes; d) finding a mathematical way to measure the interaction (the machinery of Fuzzy Logic may be a good starting point). Other possibilities include the use of topological tools such as sheaves theories [58], homotopy [59] and differential geometry.

Moreover, the mathematical structure introduced here admits further generalizations and connections with phenomenological works on conscious experience such as in $[4,5]$ and eventual generalization of other influential models of consciousness [7]. The axiomatization of different models and theories of consciousness may allow us a better understanding of their core principles, and therefore better experimental testing. It may also help to bring connections between physics, biology and psychology, avoiding the reduction to only physical descriptions.

Finally, our approach allows us to study the coupling and decoupling of the whole brain-body system and their mathematical consequences without reducing them to any particular ontology. It aligns well with embodiment $[25-27,60]$, new theoretical and experimental framework to study experience $[9,61]$, and with the recent push for formal and mathematical accounts to study conscious experience $[2-5,7,8,62]$. 


\section{Appendix}

In this supplementary section, we shortly discuss our multilayer implementation in light of its algebraic structure through Category Theory and provide some algebraic definitions used in the main text. We first introduce some content on algebraic structures used in the paper, some formal definitions on Multilayer Networks and finally categorical concepts for a formal account of multilayer networks (see $[5,12,63]$ for other introductions).

\subsection{Tensor product of Monoids}

We define the tensor product of (commutative) monoids starting from the well-known concept of cartesian product of sets: given two sets $F$ and $S$ their cartesian product is defined as

$$
F \times S=\{(f, s) / f \in F, s \in S\}
$$

We can think on the sets of first and second dishes when ordering in a restaurant: $F$ would be all available first dishes and $S$ the second ones, then $F \times S$ represent all possible combinations one can order.

Now the cartesian product of two monoids $M_{1}, M_{2}$ with internal operations $\ominus$ and $\oslash$ respectively is defined through the componentwise operation $\star$ given by

$$
(m, n) \star\left(m^{\prime}, n^{\prime}\right)=\left(m \ominus m^{\prime}, n \oslash n^{\prime}\right)
$$

for $m, m^{\prime} \in M_{1}$ and $n, n^{\prime} \in M_{2}$. Now $M_{1} \times M_{2}$ with $\star$ is a two-fold monoid.

Finally, the tensor product of monoids, written $M_{1} \otimes M_{2}$, is the quotient of their cartesian product by the relations

$$
\begin{array}{cl}
(m, n) \star\left(m^{\prime}, n\right) \sim\left(m^{\prime} \ominus m, n\right) & (m, n) \star\left(m, n^{\prime}\right) \sim\left(m, n \oslash n^{\prime}\right) \\
(\epsilon, n) \sim \epsilon \quad(m, \epsilon) \sim \epsilon
\end{array}
$$

\subsection{Partial Monoids}

Definition 17. A partial monoid is a monoid $(M, \oplus, \epsilon)$ where $\oplus$ is a partial operation. A partial monoid is said to be commutative if $\oplus$ has this property whenever defined.

That is, in a partial monoid it is true that for every $m, n \in M$ there exists $m \oplus n$ only if a certain condition is satisfied.

\subsection{Concepts on Multilayer Networks}

The following is an sketch of some of the concepts involved in the theory of Multilayer Networks belonging to [12], they are mentioned and used in the paper and given here in a more formal presentation. If a node $u$ has different aspects $a_{1}, \ldots, a_{d}$ in layers into the sets $\left(L_{1}, \ldots, L_{d}\right)$ respectively, a widely used notation is:

$$
(u, \mathbf{a}) \equiv\left(u, a_{1}, \ldots, a_{d}\right)
$$

We note here that in this paper we avoided this notation in favour of a more appropriate way to express the features we want to describe.

The set of edges is partitioned into intra-layer edges and inter-layer edges. Intra-layer edges correspond to the interactions inside layers (e.g. yellow and light blue edges, Figure 2A) and belonging to sets

$$
E_{A}=\left\{\left((u, \mathbf{a}),\left(v, \mathbf{a}^{\prime}\right)\right) \in E_{M} \mid \mathbf{a}=\mathbf{a}^{\prime}\right\}
$$

The inter-layer edges are those in $E_{C}=E_{M}-E_{A}$, which correspond to interactions between layers. 


\subsection{Category Theory}

Category theory is a branch of highly abstract mathematics. One of the main features is its power to generalize relationships across different mathematical structures. This generalization is due to its general definition of objects as mathematical types, morphisms as maps or transformations from objects/types to other objects/types, and the composition operation, which may respect associative and identity law.

\section{Category}

In short, a category, signified by $\mathfrak{C}$, consists of:

- A class of objects $o b(\mathfrak{C})$

- A set $\mathfrak{C}(A, B)$ of morphisms from $A$ to $B$, for each pair of objects $A, B$

- A composition map for each triple of objects $A, B, C$, such that

$$
\begin{array}{ccc}
\mathfrak{C}(B, C) \times \mathfrak{C}(A, B) & \longrightarrow & \mathfrak{C}(A, C) \\
(g, f) & \mapsto & g \circ f
\end{array}
$$

- For each object $A$, an identity morphism $1_{A} \in \mathfrak{C}(A, A)$

Objects, morphisms and compositions need to satisfy the following axioms:

- Associativity: for any $f \in \mathfrak{C}(A, B), g \in \mathfrak{C}(B, C), h \in \mathfrak{C}(C, D)$, there holds $(h \circ g) \circ f=h \circ(g \circ f)$;

- Identity law: for any $f \in \mathfrak{C}(A, B), 1_{B} \circ f=f=f \circ 1_{A}$.

\section{Functor}

Given categories $\mathfrak{C}$ and $\mathfrak{D}$ a functor $F: \mathfrak{C} \longrightarrow \mathfrak{D}$, consists of:

- A mapping

$$
\begin{array}{ccc}
o b(\mathfrak{C}) & \longrightarrow & o b(\mathfrak{D}) \\
A & \mapsto & F(A) ;
\end{array}
$$

- For each pair of objects $A, B$ of $\mathfrak{C}$, a map

$$
\begin{array}{ccc}
\mathfrak{C}(A, B) & \longrightarrow & \mathfrak{D}(F(A), F(B)) \\
f & \mapsto & F(f),
\end{array}
$$

Functors satisfy the following axioms:

- Preserving composition: for any morphisms $f \in \mathfrak{C}(A, B), g \in \mathfrak{C}(B, C)$, there holds $F(g \circ f)=$ $F(g) \circ F(f)$

- Preserving identity: for any object $A$ of $\mathfrak{C}, F\left(1_{A}\right)=1_{F(A)}$.

Strict monoidal category

A strict monoidal category consists of:

- A category $\mathfrak{C}$;

- A unit object $I \in o b(\mathfrak{C})$;

- A bifunctor (that is, a functor with two inputs) $-\otimes-: \mathfrak{C} \times \mathfrak{C} \longrightarrow \mathfrak{C}$,

satisfying

- Associativity: for each triple of objects $A, B, C$ of $\mathfrak{C}, A \otimes(B \otimes C)=(A \otimes B) \otimes C$; for each triple of morphisms $f, g, h$ of $\mathfrak{C}, f \otimes(g \otimes h)=(f \otimes g) \otimes h ;^{9}$

9 We define $f \otimes g: A \otimes B \longrightarrow C \otimes D$ for $f: A \longrightarrow C$ and $g: B \longrightarrow D$ as usual. 
- Unit law: for each object $A$ of $\mathfrak{C}, A \otimes I=A=I \otimes A$; for each morphism $f$ of $\mathfrak{C}, f \otimes 1_{I}=f=1_{I} \otimes f$.

A strict symmetric monoidal functor $F$ is a strict monoidal functor that preserves symmetrical structures, i.e., $F\left(\sigma_{A, B}\right)=\sigma_{F(A), F(B)}$. The definition of a general (non-strict) symmetric monoidal functor can be found in $[64]$.

\subsection{Categorizing Multilayers}

In order to give a categorical interpretation of our multilayer networks of section 3.2, we make use of the notions introduced in [13]. Following that reference, we consider a functor that produces many-coloured networks out of non-directed multigraphs.

Denote by $\mathcal{S}$ the permutation groupoid, that is, a skeleton of the groupoid of finite sets and bijections. $\mathcal{S}$ is also interpreted as the disjoint union of symmetric groups $\mathcal{S}_{n}$ where: i) objects are natural numbers, ii) all morphisms are automorphisms, and iii) the automorphism group of the object $n$ is the symmetric group $\mathcal{S}_{n} . \mathcal{S}$ is a (strict) symmetric monoidal category.

Definition 18. A one-coloured network model is a lax symmetric monoidal functor $F: \mathcal{S} \rightarrow$ Mon where Mon is the category of monoids.

Example 19. Let $M G(n)$ be the set of multigraphs on $\mathbf{n}=\{1, \ldots, n\}$. Then, a network model is a functor $M G: \mathcal{S} \rightarrow$ Mon with values $(M G(n),+)$ where + is multiset sum, that is, addition of multiplicities of edges sharing the same vertices.

The category of network models over a fixed colour set $\mathcal{C}$ is denoted by $\mathrm{NetMod}_{\mathcal{C}}$ (see [13]). NetMod is a symmetric monoidal category and therefore we can tensor functors such as those introduced above.

Definition 20. A network model for multigraphs with coloured edges is a functor

$$
M G^{\otimes \mathcal{C}}: \mathcal{S} \rightarrow \text { Mon }
$$

where $M G^{\otimes \mathcal{C}}(n)$ is a product of $|\mathcal{C}|$ copies of the monoid $M G(n)$, being $|\mathcal{C}|$ the cardinal of the set $\mathcal{C}$.

It is crucial that, in these tensor models, a network is not only a $\mathcal{C}$-tuple of multigraphs: it may also be viewed as a multigraph with as many edges of each colour between any pair of distinct vertices as they appear in every layer. The fact that the functor $M G^{\otimes \mathcal{C}}$ is indeed a network model comes from the disjoint union operation:

$$
M G^{\otimes \mathcal{C}}(n) \times M G^{\otimes \mathcal{C}}(m) \stackrel{\sqcup}{\longrightarrow} M G^{\otimes \mathcal{C}}(n+m)
$$

The particular kind of network model considered for the rotation multigraphs of section 5 depends precisely on the rotation angle and, subsequently, a variable on time. However, it does not satisfy one of the requirements introduced in [13], namely, being lax monoidal (see [13]). This is due to the fact that the operation analogous to $\sqcup$ in non-rotation models, turns out to be partial in the rotation ones (i.e. subject to the condition $|\alpha(t)|+|\beta(t)| \geq \pi / 2$ ). Since we are interested in the interaction among layers, every rotation concept is defined under the assumption that that interaction does take place.

Definition 21. Given $t \in T$ and PMon the category of partial monoids, a network rotation model is a functor

$$
R M G^{t}: \mathcal{S} \rightarrow \text { PMon }
$$

Example 22. A rotation network model for multigraphs with coloured edges is a functor

$$
\left[R M G^{t}\right]^{\otimes \mathcal{C}}: \mathcal{S} \rightarrow \text { PMon }
$$

where $\left[R M G^{t}\right]^{\otimes \mathcal{C}}(n)$ is a product of $|\mathcal{C}|$ partial monochrome monoids $R M G^{t}(n)$ (recall that $\mathcal{C}$ can be a multiset and then $|\mathcal{C}|$ refers to the multiset cardinal). 


\subsection{Distributivity}

The $\odot$ operation is distributive over $\times$. That is, we obtain:

$$
\left(\prod_{i=1}^{d_{1}} G_{i}\right) \odot\left(\prod_{j=1}^{d_{2}} H_{j}\right)=\prod_{i, j}\left(G_{i} \odot H_{j}\right)=G_{1} \odot H_{1} \times \ldots \times G_{d_{1}} \odot H_{d_{2}}
$$

whenever we calculate over expressions of multilayers containing monochrome layers such as

$$
\begin{gathered}
G_{1} \times \ldots \times G_{d_{1}} \in M G^{c_{1}}\left(n_{1}\right) \times \ldots \times M G^{c_{d_{1}}}\left(n_{d_{1}}\right) \\
H_{1} \times \ldots \times H_{d_{2}} \in M G^{c^{\prime}}{ }_{1}\left(m_{1}\right) \times \ldots \times M G^{c^{\prime} d_{2}}\left(m_{d_{2}}\right)
\end{gathered}
$$

The expression 1 contains all combinations in the form $G_{i} \odot H_{j}$ for $i=1, \ldots, d_{1}$ and $j=1, \ldots, d_{2}$ and lives in

$$
M G^{c_{1} \otimes c^{\prime}}{ }_{1}\left(n_{1}+m_{1}-p_{11}\right) \times \ldots \times M G^{c_{d_{1}} \otimes c^{\prime} d_{2}}\left(n_{d_{1}}+m_{d_{2}}-p_{d_{1} d_{2}}\right)
$$

where $p_{i j}=\left|V\left(G_{i}\right) \cap V\left(H_{j}\right)\right|$.

\subsection{Inclusion-Exclusion Principle}

Counting the number of vertices into a graph

$$
\left[G_{1}, \alpha_{1}\right] \odot \ldots \odot\left[G_{k}, \alpha_{k}\right]
$$

means considering the known as Inclusion-Exclusion Principle in the form:

$$
\begin{gathered}
\sum_{1 \leq i_{1} \leq k}\left|V\left(G_{i_{1}}\right)\right|-\sum_{1 \leq i_{1} \leq i_{2} \leq k}\left|V\left(G_{i_{1}}\right) \cap V\left(G_{i_{2}}\right)\right|+\sum_{1 \leq i_{1} \leq i_{2} \leq i_{3} \leq k}\left|V\left(G_{i_{1}}\right) \cap V\left(G_{i_{2}}\right) \cap V\left(G_{i_{3}}\right)\right|-\ldots \\
\ldots+(-1)^{k+1}\left|\bigcap_{i=1}^{k} V\left(G_{i}\right)\right|
\end{gathered}
$$

As done in section 4.2 we generalize $\odot$ to longer expressions such as

$$
\left(\prod_{i=1}^{k_{1}}\left[G_{i}, \alpha_{i}\right]\right) \odot\left(\prod_{j=1}^{k_{2}}\left[H_{j}, \beta_{j}\right]\right)=\left[G_{p_{1}}, \alpha_{p_{1}}\right] \odot\left[H_{q_{1}}, \beta_{q_{1}}\right] \times \ldots \times\left[G_{p_{l}}, \alpha_{p_{l}}\right] \odot\left[H_{q_{m}}, \beta_{q_{m}}\right]
$$

making use of the distributivity of $\odot$ and where the products $[G, \alpha] \odot[H, \beta]$ in equation 2 are those formed by interacting pairs $[G, \alpha],[H, \beta]$ with

$$
\left\{\left[G_{p_{1}}, \alpha_{p_{1}}\right], \ldots,\left[G_{p_{l}}, \alpha_{p_{l}}\right]\right\} \subseteq\left\{\left[G_{1}, \alpha_{1}\right], \ldots,\left[G_{d_{1}}, \alpha_{d_{1}}\right]\right\}
$$

and

$$
\left\{\left[H_{q_{1}}, \beta_{q_{1}}\right], \ldots,\left[H_{q_{m}}, \beta_{q_{m}}\right]\right\} \subseteq\left\{\left[H_{1}, \beta_{1}\right], \ldots,\left[H_{d_{2}}, \beta_{d_{2}}\right]\right\}
$$

Equation 2 is then seen as a simultaneous interaction among layers.

\subsection{Decomposition in Biobrane}

The decomposition of graphs in Biobrane is determined by the equation:

$$
\bigodot_{j=1}^{m}\left[G_{j}, \alpha_{j}\right]=\cup_{j=1}^{m}\left[H_{j}, \beta_{j}\right]
$$

where we impose that $E\left(G_{j}\right)=E\left(H_{j}\right)$ for $j=1, \ldots m$ but $\left|V\left(H_{j}\right)\right|=n$ and, moreover, $\left|\beta_{j}\right|+\left|\beta_{l}\right|<\pi / 2$ for $j, l=1, \ldots, m$. 
$H_{j}$ are then defined exactly as $G_{j}$ but adding an isolated vertice for every missing one in $G_{j}$ up to n. $\left[G_{j}, \alpha_{j}\right]$ are the monochrome graphs in Biobrane from which $[G, \alpha]$ was constructed and $\left[H_{j}, \beta_{j}\right]$ are defined from them satisfying the following conditions:

- every $\left[H_{j}, \beta_{j}\right]$ is monochromatic

- every $\left[H_{j}, \beta_{j}\right]$ has $n$ vertices

- $m$ is the number of layers used to build and decompose $[G, \alpha]$ by applications of $\odot$ and $\cup$ respectively

- $\left|\beta_{j}\right|+\left|\beta_{l}\right|<\pi / 2$ for $j, l=1, \ldots, m$

- $m_{1}+\ldots+m_{d}=m$.

Author Contributions: Both authors contributed equally to this article. Specifically, CMS conceptualized the model, reviewed empirical data, wrote original and edited versions, while JDB formalized the model, reviewed mathematical state of the art, wrote original and edited versions.

Funding: This research was partially funded by Comisión Nacional de Investigación Ciencia y Tecnología (CONICYT, currently ANID) through Programa Formacion de Capital Avanzado (PFCHA), Doctoral scholarship Becas Chile: CONICYT PFCHA/DOCTORADO BECAS CHILE/2016 - 72170507. CMS also acknowledge the grant: Categorical Theories of Consciousness: Bridging Neuroscience and Fundamental Physics, FQXi-RFP-CPW-2018.

Acknowledgments: The authors appreciate early discussions from Giovanni Rabuffo, Xerxes Arsiwalla, Moritz Kriegleder, Sean Tull and Daniel Meling in the modelling consciousness workshop 2019 (Austria), as well as the organizers of the modelling of consciousness conference 2019 (Oxford), where some preliminary ideas were presented. The authors also appreciate comments on the first drafts given by Bob Coecke and the anonymous reviewers at ACT 2020 conference and the especial issue of models of consciousness at entropy 2020, during early stages of this work. Finally, the authors thanks comments from Matthieu Koroma in the last versions of this work.

Conflicts of Interest: The authors declare no conflict of interest.

\section{References}

1. AMCS. Association for Mathematical Consciousness Science (AMCS). https://amcs-community.org 2021.

2. Yoshimi, J. Mathematizing phenomenology. Phenomenology and the Cognitive Sciences 2007, 6, 271-291. doi:10.1007/s11097-007-9052-4.

3. Prentner, R. Consciousness and topologically structured phenomenal spaces. Consciousness and Cognition 2019, 70, 25-38. doi:10.1016/j.concog.2019.02.002.

4. Signorelli, C.M.; Wang, Q.; Khan, I. A Compositional Model of Consciousness Based on Consciousness-Only. Entropy 2021, 23, 308, [2007.16138v2]. doi:10.3390/e23030308.

5. Signorelli, C.M.; Wang, Q.; Coecke, B. Reasoning about conscious experience with axiomatic and graphical mathematics. Consciousness and Cognition 2021, 95, 103168. doi:10.1016/j.concog.2021.103168.

6. Oizumi, M.; Albantakis, L.; Tononi, G. From the phenomenology to the mechanisms of consciousness: Integrated Information Theory 3.0. PLoS computational biology 2014, 10, e1003588. doi:10.1371/journal.pcbi.1003588.

7. Tull, S.; Kleiner, J. Integrated Information in Process Theories. Proceedings of SEMSPACE 2020, 2020, pp. 1-22, [2002.07654].

8. Tsuchiya, N.; Saigo, H. Applying Yoneda's lemma to consciousness research: categories of level and contents of consciousness. Preprint 2020. doi:10.31219/osf.io/68nhy.

9. Signorelli, C.M.; Meling, D. Towards new concepts for a biological neuroscience of consciousness. Cognitive Neurodynamics 2021, 15, 783-804. doi:10.1007/s11571-020-09658-7.

10. Varela, F.J.; Thompson, E.; Rosch, E. The Embodied Mind, revised ed ed.; MIT Press, 2016; p. 392.

11. Thompson, E.; Varela, F. Radical embodiment: neural dynamics and consciousness. Trends in cognitive sciences 2001, 5, 418-425.

12. Kivela, M.; Arenas, A.; Barthelemy, M.; Gleeson, J.P.; Moreno, Y.; Porter, M.A. Multilayer networks. Journal of complex networks 2014, 2, 203-271. doi:10.1093/oso/9780198809456.003.0001.

13. Baez, J.C.; Foley, J.; Moeller, J.; Pollard, B.S. Network models, 2018, [1711.00037].

14. MacLane, S. Categories for the Working Mathematician, second ed.; Springer New York, 1998. doi:10.1007/978-1-4757-4721-8.

15. Mashour, G.A.; Roelfsema, P.; Changeux, J.P.; Dehaene, S. Conscious Processing and the Global Neuronal Workspace Hypothesis. Neuron 2020, 105, 776-798. doi:10.1016/j.neuron.2020.01.026. 
16. Tononi, G.; Boly, M.; Massimini, M.; Koch, C. Integrated information theory: from consciousness to its physical substrate. Nature reviews. Neuroscience 2016, 17, 450-61. doi:10.1038/nrn.2016.44.

17. Boccaletti, S.; Bianconi, G.; Criado, R.; del Genio, C.I.; Gómez-Gardeñes, J.; Romance, M.; Sendiña-Nadal, I.; Wang, Z.; Zanin, M. The structure and dynamics of multilayer networks. Physics Reports 2014, 544, 1-122, [1407.0742]. doi:10.1016/j.physrep.2014.07.001.

18. Signorelli, C.M.; Szczotka, J.; Prentner, R. Explanatory profiles of models of consciousness - towards a systematic classification. Neuroscience of Consciousness 2021, 2021. doi:10.1093/nc/niab021.

19. Chalmers, D. The puzzle of conscious experience. Scientific American 1995, 273, 80-86.

20. Thomas Nagel. What is it like to be a bat? The Philosophical Review 1974, 83, 435-450.

21. Husserl, E. General Introduction to a Pure Phenomenology; Martinus Nijhoff Publishers, 1983.

22. Merleau-Ponty, M. Phenomenology of perception; Routledge: London, 2005.

23. Varela, F.J. Neurophenomenology: A Methodological Remedy for the Hard Problem. Journal of Consciousness Studies 1996, 3, 330-349.

24. Thompson, E. Life and mind: From autopoiesis to neurophenomenology. A tribute to Francisco Varela. Phenomenology and the Cognitive Sciences 2004, 3, 381-398. doi:10.1023/b:phen.0000048936.73339.dd.

25. Thompson, E. Mind in Life; Harvard University Press: Cambridge, Massachusetts, 2007.

26. Thompson, E. Waking, Dreaming, Being; Columbia University Press, 2014; p. 496.

27. Gallagher, S.; Zahavi, D. The phenomenological mind, first ed.; Routledge: London, 2008. doi:10.5860/choice.50-1996.

28. De Jaegher, H.; Di Paolo, E.; Gallagher, S. Can social interaction constitute social cognition? Trends in Cognitive Sciences 2010, 14, 441-447. doi:10.1016/j.tics.2010.06.009.

29. Di Paolo, E.; Buhrmann, T.; Barandiaran, X. Sensorimotor Life. An enactive proposal; Oxford University Press, 2017; p. 304.

30. Lawvere, F.W.; Schanuel, S.H. Conceptual mathematics: a first introduction to categories, second ed.; Cambridge University Press, 2009; p. 410.

31. Riehl, E. Infinite Math. Scientific American 2021, 325, 32-41. doi:10.1038/scientificamerican1021-32.

32. Rescher, N. Process Philosophy. Stanford Encyclopedia of Philosophy 2012.

33. Whitehead, A.N. Process and Reality: an Essay in Cosmology; Free Press: New York, 1929.

34. Coecke, B., Ed. New Structures for Physics, lectures n ed.; Vol. 813, Lecture Notes in Physics, Springer Berlin Heidelberg: Berlin, Heidelberg, 2011; p. 1034. doi:10.1007/978-3-642-12821-9.

35. Coecke, B.; Duncan, R.; Kissinger, A.; Wang, Q. Generalised Compositional Theories and Diagrammatic Reasoning. In Quantum Theory: Informational Foundations and Foils. Fundamental Theories of Physics.; Chiribella, G.; Spekkens, R., Eds.; Springer, 2016; Vol. 181, pp. 309-366, [1506.03632]. doi:10.1007/978-94-017-7303-4_10.

36. Coecke, B. Compositionality as we see it, everywhere around us 2021. [2110.05327].

37. Awodey, S. Category Theory, first ed.; Oxford University Press: Oxford, 2006; p. 266.

38. Tripathi, V.; Bharadwaj, P. Neuroscience of the yogic theory of consciousness. Neuroscience of consciousness 2021, 7, 1-15. doi:10.1093/nc/niab030.

39. Tagliazucchi, E.; Crossley, N.; Bullmore, E.T.; Laufs, H. Deep sleep divides the cortex into opposite modes of anatomical-functional coupling. Brain Structure and Function 2016, 221, 4221-4234. doi:10.1007/s00429-015-1162-0.

40. Fox, M.D.; Snyder, A.Z.; Vincent, J.L.; Corbetta, M.; Van Essen, D.C.; Raichle, M.E. The human brain is intrinsically organized into dynamic, anticorrelated functional networks. Proceedings of the National Academy of Sciences 2005, 102, 9673-9678, [NIHMS150003]. doi:10.1073/pnas.0504136102.

41. Paolo, E.A.D.; Thompson, E.; Beer, R.D. Laying down a forking path : Incompatibilities between enaction and the free energy principle. pp. 1-43.

42. Varela, F.; Maturana, H.; Uribe, R. Autopoiesis: The organization of living systems, its characterization and a model. BioSystems 1974, 5, 187-196.

43. Varela, F. Principles of Biological Autonomy; Elsevier North Holland: New York, 1979; p. 306.

44. Maturana, H.; Varela, F. De máquinas y seres vivos, quinta ed.; Editorial Universitaria S.A.: Santiago de Chile, 1998; p. 136.

45. Mesulam, M. Large-Scale Neurocognitive Networks and Distributed Processing for Attention, Language, and Memory. Neurological Progress. Annals of Neurology 1990, 28, 597'613. doi:10.1002/ana.410280502. 
46. Pessoa, L. Understanding brain networks and brain organization. Physics of Life Reviews 2014, 11, 400-435, [1403.7151]. doi:10.1016/j.plrev.2014.03.005.

47. de Barros, J.A.; Montemayor, C.; De Assis, L.P. Contextuality in the Integrated Information Theory. Quantum Interactions 2016; de Barros, J.A., Ed. Springer International Publishing, 2017, Vol. 8369, pp. 57-70, [1408.5697]. doi:10.1007/978-3-642-54943-4.

48. Kleiner, J.; Hoel, E. Falsification and consciousness. Neuroscience of Consciousness 2021, 2021, 1-15, [2004.03541]. doi:10.1093/nc/niab001.

49. Baars, B.J. Global workspace theory of consciousness: toward a cognitive neuroscience of human experience. Progress in brain research 2005, 150, 45-53. doi:10.1016/S0079-6123(05)50004-9.

50. Dehaene, S.; Changeux, J.P. Experimental and theoretical approaches to conscious processing. Neuron 2011, 70, 200-27. doi:10.1016/j.neuron.2011.03.018.

51. Tononi, G.; Edelman, G.M. Consciousness and complexity. Science 1998, 282, $1846-1851$. doi:10.1126/science.282.5395.1846.

52. Ehresmann, A.C.; Vanbremeersch, J.P. MENS, a mathematical model for cognitive systems. Journal of Mind Theory 2009, 0, 129-180. doi:10.1021/ja042798i.

53. Ward, A.F.; Wegner, D.M. Mind-blanking: When the mind goes away. Frontiers in Psychology 2013, 4, 1-15. doi:10.3389/fpsyg.2013.00650.

54. Naccache, L. Why and how access consciousness can account for phenomenal consciousness. Philosophical Transactions of the Royal Society B: Biological Sciences 2018, 373. doi:10.1098/rstb.2017.0357.

55. Demertzi, A.; Tagliazucchi, E.; Dehaene, S.; Deco, G.; Barttfeld, P.; Raimondo, F.; Martial, C.; Fernández-Espejo, D.; Rohaut, B.; Voss, H.U.; Schiff, N.D.; Owen, A.M.; Laureys, S.; Naccache, L.; Sitt, J.D. Human consciousness is supported by dynamic complex patterns of brain signal coordination. Science Advances 2019, 5, 1-11. doi:10.1126/sciadv.aat7603.

56. Rodríguez, E. Ideas para naturalizar el estudio de la conciencia. In Ciencias de la mente: aproximaciones desde Latinoamerica; Kronmüller, E.; Cornejo, C., Eds.; Editorial Granica, 2008; pp. 301-324.

57. Searle, J.R. Consciousness, free action and the brain. Journal of Consciousness Studies 2000, 7, 3-22.

58. Abramsky, S. Contextuality: At the Borders of Paradox. In Categories for the Working Philosopher; Landry, E., Ed.; Oxford University Press, 2018; Vol. 1, pp. 1-471, [arXiv:1011.1669v3]. doi:10.1093/oso/9780198748991.001.0001.

59. Grigor'yan, A.; Muranov, Y.; Vershinin, V.; Yau, S.T. Path homology theory of multigraphs and quivers. Forum Mathematicum 2018, 30, 1319-1337. doi:10.1515/forum-2018-0015.

60. Gallagher, S. How the Body Shapes the Mind; Oxford University Press, 2006; pp. 1-294. doi:10.1093/0199271941.001.0001.

61. Signorelli, C.M.; Diaz Boils, J.; Tagliazucchi, E.; Jarraya, B.; Deco, G. From brain-body function to consciousness interaction. (under review) 2021.

62. Kleiner, J. Mathematical Models of Consciousness. Entropy 2020, 22, 609, [arXiv:1907.03223v1]. doi:10.3390/e22060609.

63. Fong, B.; Spivak, D.I. An Invitation to Applied Category Theory; Cambridge University Press, 2019 ; p. 348. doi:10.1017/9781108668804.

64. Maclane, S. Categorical Algebra. Bulletin of the American Mathematical Society 1965, 71, 40-106. doi:10.1090/S0002-9904-1965-11234-4. 